\title{
Sensitivity and Negative Predictive Value of Motor Evoked Potentials of the Facial Nerve
}

\author{
Nicolas Bovo ${ }^{1}$ Shahan Momjian ${ }^{2}$ Renato Gondar ${ }^{3}$ \\ ${ }^{1}$ Neurochirurgie, Université de Genève Centre Médical Universitaire, \\ Geneva, Switzerland \\ ${ }^{2}$ Hôpitaux Universitaires de Genève, Geneva, Switzerland \\ ${ }^{3}$ Division of Neurosurgery, Neurosciences Cliniques, Hôpitaux \\ Universitaires de Genève, Geneva, Switzerland \\ ${ }^{4}$ Department of Neurosurgery, Hôpitaux Universitaires de Genève, \\ Geneva, Switzerland
}

J Neurol Surg A Cent Eur Neurosurg 2021;82:317-324.

\section{Philippe Bijlenga $^{2}$ Karl Schaller ${ }^{4}$ Colette Boëx ${ }^{2}$}

\author{
Address for correspondence Colette Boëx, PhD, Department of \\ Neurology, University Hospital of Geneva, rue Gabrielle-Perret \\ Gentil 4, Geneva 1211, Switzerland (e-mail: Colette.Boex@hcuge.ch).
}

\begin{abstract}
Keywords

- intraoperative neuromonitoring

- facial nerve

- cerebellopontine angle

- motor evoked potentials

- vestibular schwannomas

Objective The objective of this study was to determine the performance of the standard alarm criterion of motor evoked potentials (MEPs) of the facial nerve in surgeries performed for resections of vestibular schwannomas or of other lesions of the cerebellopontine angle.

Methods This retrospective study included 33 patients (16 with vestibular schwannomas and 17 with other lesions) who underwent the resection surgery with transcranial MEPs of the facial nerve. A reproducible 50\% decrease in MEP amplitude, resistant to a $10 \%$ increase in stimulation intensity, was applied as the alarm criterion during surgery. Facial muscular function was clinically evaluated with the HouseBrackmann score (HBS), pre- and postsurgery at 3 months.

Results In the patient group with vestibular schwannoma, postoperatively, the highest sensitivity and negative predictive values were found for a $30 \%$ decrease in MEP amplitude, that is, a criterion stricter than the $50 \%$ decrease in MEP amplitude criterion, prone to trigger more warnings, used intraoperatively. With this new criterion, the sensitivity would be $88.9 \%$ and the negative predictive value would be 85.7\%. In the patient group with other lesions of the cerebellopontine angle, the highest sensitivity and negative predictive values were found equally for 50,60 , or $70 \%$ decrease in MEP amplitude. With these criteria, the sensitivities and the negative predictive values would be $100.0 \%$.

Conclusion Different alarm criteria were found for surgeries for vestibular schwannomas and for other lesions of the cerebellopontine angle. The study consolidates the stricter alarm criterion, that is, a criterion prone to trigger early warnings, as found previously by others for vestibular schwannoma surgeries (30\% decrease in MEP amplitude).
\end{abstract}

received

March 5, 2019

accepted after revision

December 20, 2019

published online

January 21, 2021 (c) 2021. Thieme. All rights reserved.

Georg Thieme Verlag KG,

Rüdigerstraße 14,

70469 Stuttgart, Germany
DOI https://doi.org/

10.1055/s-0040-1719026. ISSN 2193-6315. 


\section{Introduction}

Neurosurgeries undertaken for the resection of vestibular schwannomas ${ }^{1,2}$ or of other cerebellopontine angle lesions such as meningiomas, ${ }^{3}$ or for trigeminal neuralgia ${ }^{4}$ or hemifacial spasms ${ }^{5}$ can be complicated by deterioration of the facial nerve function. In all cases, not only the proximity of the lesion to the nerve but also the size of the lesion increase the risk of facial nerve injuries. ${ }^{6}$

For these surgeries, intraoperative monitoring techniques applied to the facial nerve have been developed using three different modalities: electromyography (EMG), direct electrical stimulation, and motor evoked potentials (MEPs).

EMG recording has been applied for continuous monitoring of the nerve activity ${ }^{7-9}$ and analyzed with the objective of predicting the postoperative functions of the facial nerve. ${ }^{10,11}$ EMG has been applied in our center as a background monitoring technique of the possible mechanical stimulations of the nerve only.

Direct electrical stimulation of the facial nerve, introduced in otolaryngology ${ }^{12,13}$ and neurosurgery, ${ }^{14}$ helps comfort the location of the facial nerve throughout the surgery. It has been acknowledged to be effective in limiting the risks of postoperative facial paresis. ${ }^{15,16}$

MEPs of the facial nerve help check the integrity of the motor pathway from the primary motor cortex to the muscles of the contralateral hemiface. The efficacy of MEPs is dependent on the alarm criterion applied to trigger an alarm and the consecutive adaptation of the surgical strategy to prevent an irreversible damage to the facial nerve. The choice of the alarm criterion is still a matter of debate. A 50\% decrease in MEP amplitude has been reported as reliable in some studies ${ }^{17,18}$ but not strict enough in others. $^{19-21}$

In this context, the objective of this retrospective study was to analyze the validity of the alarm criterion applied at the time of surgery, that is, a reproducible $50 \%$ decrease in MEP amplitude in neurosurgeries performed for vestibular schwannomas or for other lesions of the cerebellopontine angle.

Although intraoperative monitoring should be considered an "intervention"-like technique and hence its efficacy ideally evaluated with control groups, as discussed by Howick et $\mathrm{al}^{22}$ this is actually not performed for ethical considerations. In addition, as discussed by Howick et al, ${ }^{22}$ intraoperative monitoring cannot present false-positive cases. This point is of major importance. Indeed, in case an alarm has been raised and no new deficit was observed postoperatively, it cannot be determined if that was due to or not due to the alarm raised during the surgery. Hence, specificity and positive predictive value, both necessitating the identification of false-positive cases, should not be calculated for intraoperative neuromonitoring techniques and in particular for MEPs. The computation of sensitivity and negative predictive values for MEPs remains effective to postoperatively evaluate an alarm criterion, in the single objective to avoid positive cases in the procedures to come.

\section{Methods}

\section{Patients}

This retrospective study included patients who undertook a surgery of the cerebellopontine angle, with corticobulbar MEPs in our center, between 2015 and 2018. The participation to the study was offered to all patients who undertook such a surgery. They all gave their signed agreement to be part of this study anonymously. The local ethics committee approved this study (CCER, 2018-02029).

Thirty-three patients participated in the study: 16 patients suffered from a vestibular schwannoma ( 7 males, 9 females; mean age 53 years, standard deviation 11.5 years), while 17 patients suffered from various other cerebellopontine angle lesions (5 meningiomas, 4 hemifacial spasms, 6 trigeminal neuralgias, 1 epidermoid cyst, and 1 dural arteriovenous fistula; 7 males, 10 females; mean age 59 years, standard deviation 13.4 years). These lesions were reached by a retrosigmoid approach.

\section{Anesthesia}

General anesthesia was performed with propofol and sufentanil: induction was reached with propofol concentrations of 4.5 to $5.0 \mathrm{mg} / \mathrm{mL}$ and sufentanil concentrations of 0.3 to $0.4 \mathrm{ng} / \mathrm{mL}$. Concentrations were then adjusted throughout the operation according to the needs of the patient. No inhaled agents were used during the resection.

\section{Monitoring}

The facial MEPs were performed with transcranial electrical stimulation (train of 4 anodic pulses, $400 \mathrm{~Hz}, 0.4-\mathrm{ms}$ phase duration, no averaging; NimEclipse system, Medtronic, Minneapolis, Minnesota, United States). One subdermal anode electrode was placed either between $\mathrm{C} 5$ and $\mathrm{C} 3$ or between C6 and $\mathrm{C}^{23}$ (international 10/10 EEG system) on the contralateral side of the facial nerve to monitor, with the cathode at the vertex (DME1001, Medtronic Xomed Inc., Jacksonville, Florida, United States). The location of anodes was therefore approximately similar to that of the anodes already reported for MEPs of the facial nerve. ${ }^{23}$ Stimulation made of a single pulse was applied from time to time to verify that the stimulation did not directly excite the facial nerve distally. ${ }^{24}$

The MEPs were measured using subdermal needles (DSN2282, Medtronic Xomed Inc., Jacksonville, Florida, United States) positioned in different muscles of the lower hemiface (mentalis or orbicularis oris, nasalis) ipsilateral to the side of the surgery. MEPs from the ipsilateral hand (thenar muscles) were also recorded to detect possible changes in systemic parameters, such as changes in mean blood pressure. MEPs were filtered (bandpass: $80-2,000 \mathrm{~Hz}$ ) to improve their reproducibility, attenuating the low-frequency part of the MEP signal.

During surgery, the alarm criterion was defined as a reproducible 50\% decrease in MEP amplitude, resistant to a $10 \%$ increase in stimulation intensity.

Direct electrical stimulation was applied using a monopolar probe (Inomed Medizintechnik $\mathrm{GmbH}$, Emmendingen, Germany). Each stimulation consisted of a biphasic pulse of $0.2 \mathrm{~ms}$ per phase, with maximum intensity of $0.7 \mathrm{~mA}$, delivered every second. Motor responses were collected for the same 
ipsilateral muscles (mentalis or orbicularis oris, nasalis) and for the frontalis muscle.

\section{Facial Outcome}

To correlate electrophysiological measurements with the clinical outcome, the $\mathrm{HBS}^{25}$ measuring facial paresis was applied. The scores were evaluated preoperatively and 7 days and 3 months postoperatively at the time of standard clinical consultations. This score is graded from 1 to 6 , with 1 being normal facial function and 6 a total facial nerve palsy. It includes the movements of the forehead, the eyes, and the mouth.

\section{Statistics}

The patients were classified into different categories according to the possible changes in the amplitude of facial MEPs and the possible postoperative changes in their HBS. The postoperative distribution of the cases among true-positive (TP; alarm raised, reproducible 50\% decrease in MEP amplitude, resistant to a $10 \%$ increase in stimulation intensity, at the end of monitoring and new deficits), true-negative (TN; alarm raised or not at the time of surgery, decrease in MEP amplitude of $<50 \%$ or none at the end of monitoring and no deficit), and false-negative (FN; alarm raised or not at the time of surgery, decrease in MEP amplitude of $<50 \%$ or none at the end of monitoring but new deficits) cases were computed for both groups of patients.

The confidence intervals of sensitivity and negative predictive values were calculated using the MedCalc software (https://www.medcalc.org/calc/diagnostic_test.php).

\section{Results}

Facial MEPs were obtained in 31 out of 33 patients. Facial MEPs could not be obtained without concomitant distal stimulation of the facial nerve in two patients. In one patient with hemifacial spasm, the HBS was unreliable in the context of preoperative Botox treatment.

The facial MEPs obtained in patient P10 are described in -Fig. 1. The patient suffered from a left vestibular schwannoma (Koos grade III), with intrapetrous extension into the middle and internal ear, and with preoperative cophosis. The MEP loss (white arrow in - Fig. 1) occurred while MEPs were interrupted for direct electrical stimulation during intradural resection. Resection was stopped at that time, leaving small residue. Note that although every MEP trace in - Fig. 1 is the response to one single stimulation obtained without averaging responses, the MEP amplitudes of the orbicularis oris muscle ("OrOr +-OrOr-") were reproducible. This could be obtained thanks to filters (80-

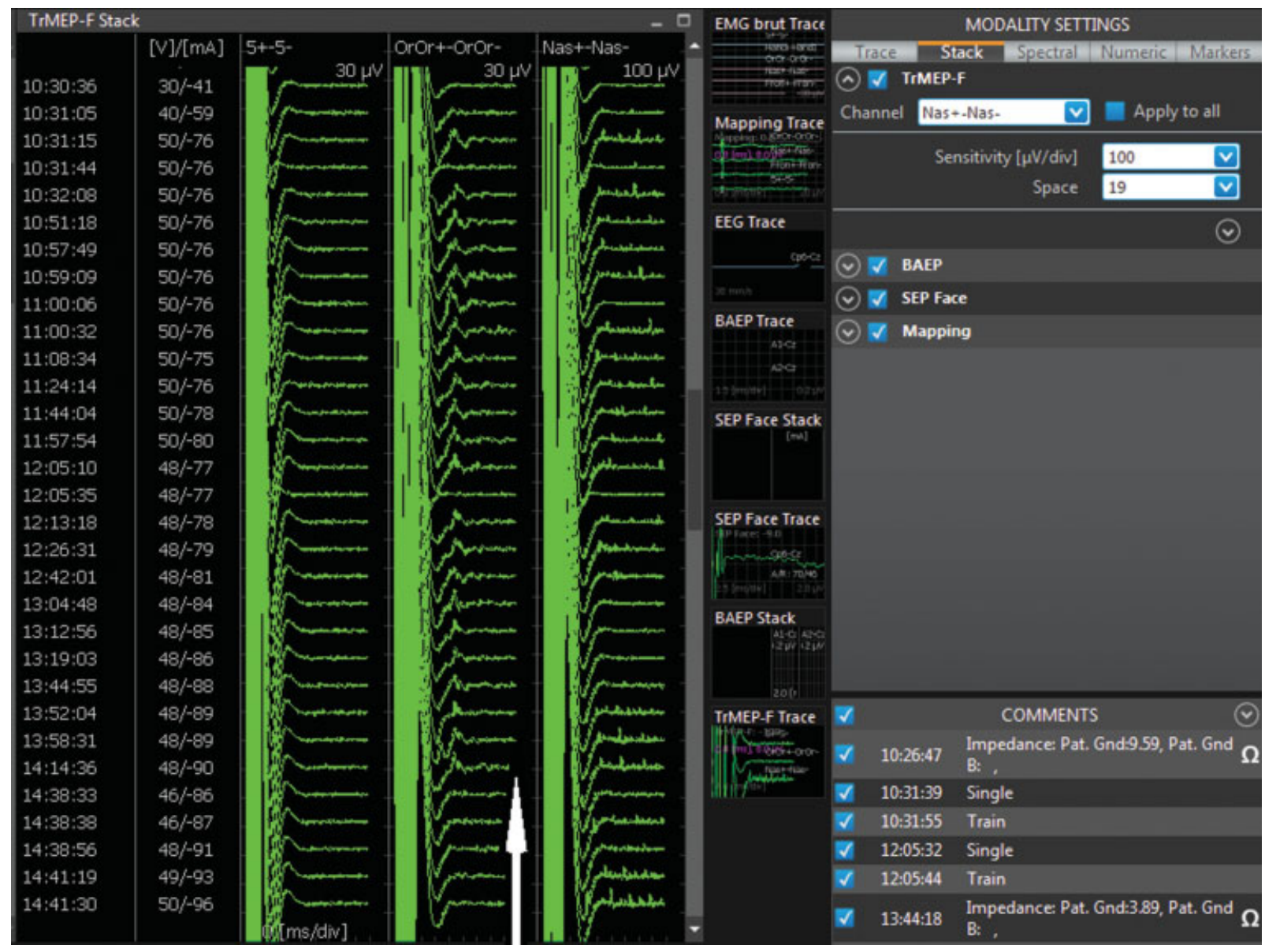

Fig. 1 Facial motor evoked potentials (MEP) (filtering $80-1,500 \mathrm{~Hz}$ ) recorded in patient P10 during resection of a vestibular schwannoma. The reproducible loss $(-100 \%)$ of the orbicularis oris MEP was resistant to an $8 \%$ increase in stimulation intensity (white arrow). Three months postsurgery, the patient did not yet recover from this new facial paresis. 


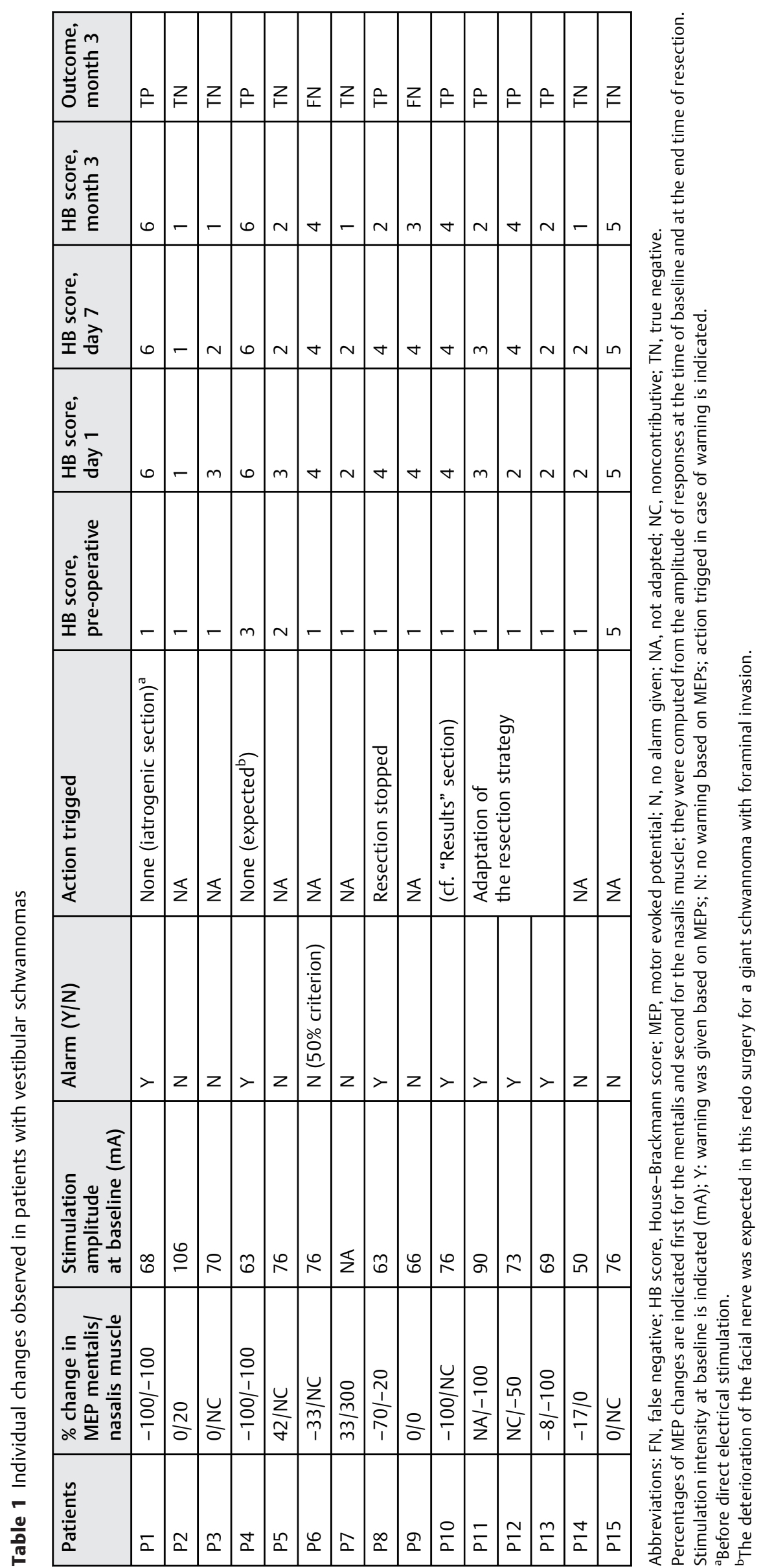


$1,500 \mathrm{~Hz}$ ) applied to each MEP to improve the signal-to-noise ratio.

Among the group of 15 patients with vestibular schwannomas and with contributive facial MEPs (-Table 1), at 3 months postsurgery, no change in HBS was observed in 6 patients for whom no change in MEP amplitude or a decrease in MEP amplitude of $<50 \%$ was observed at the time of surgery (6 TN); an increase in the HBS was observed in 7 patients for whom a reproducible decrease of $>50 \%$ in MEP amplitude, resistant to a $10 \%$ increase in stimulation intensity, was observed (7 TP); an increase in HBS was observed in 2 patients for whom no decrease in MEP amplitude or a decrease of $<50 \%$ in MEP amplitude was observed (2 FN). - Table 2 indicates sensitivities and negative predictive values computed postoperatively for different percentages of decrease in MEP amplitudes, in the group of patients with vestibular schwannomas. With the standard alarm criterion of 50\% decrease in MEP amplitude, at 3 months postoperatively, facial MEPs presented a sensitivity of $77.8 \%$ (confidence interval $[\mathrm{CI}]:$ 40.0-97.2\%) and a negative predictive value of $75.0 \%$ (CI: 46.0-91.3\%). Postoperative analyses suggest better sensitivity of $88.9 \%$ (CI: 51.7-99.7\%) and negative predictive value of $85.7 \%$ (CI: $48.6-97.4 \%$ ) with an alarm criterion of $30 \%$ decrease in the MEP amplitude.

Among the group of 15 patients with other cerebellopontine angle lesions and with contributive facial MEPs (-Table 3), at 3 months postsurgery, no change in HBS was observed in 14 patients for whom no change in MEP amplitude or no decrease in MEP amplitude of $>75 \%$ was observed at the time of surgery (14 TN). A deterioration in the HBS of one grade was observed in one patient for whom a $75 \%$ decrease in MEP amplitude was observed (1 TP).

- Table 4 indicates sensitivities and negative predictive values computed postoperatively for different percentages of decrease in MEP amplitudes, in the group of patients with other cerebellopontine angle lesions. With the standard alarm criterion of $50 \%$ and a $70 \%$ decrease in MEP amplitude as alarm criterion, at 3 months postoperatively, facial MEPs presented a sensitivity of $100.0 \%$ (CI: $2.5-100.0 \%$ ) and a negative predictive value of $100.0 \%$. Nevertheless, given the small size of the group with other cerebellopontine angle lesions, the accepted 50\% decrease in MEP amplitude as alarm criterion should not be loosened.

\section{Discussion}

The standard alarm criterion of 50\% decrease in MEP amplitude did not appear reliable for the monitoring of the facial nerve in all cerebellopontine angle surgeries. It appears that different alarm criteria should be used in the monitoring for patients undergoing vestibular schwannoma surgery and for patients with other cerebellopontine angle lesions. Variations in facial MEPs observed during vestibular schwannoma resections must be carefully monitored because a reproducible decrease of $30 \%$ in MEP amplitude, resistant to a $10 \%$ increase in stimulation intensity, can already be assigned to a long-term facial nerve dysfunction. This observation may be due to the fact that vestibular schwannomas are most often intertwined with fibers of the facial nerve. This is similar to the application of different alarm criteria for transcranial as opposed to direct cortical stimulation during the resection of insular, pre-, or postcentral lesions. ${ }^{26}$ Dong et $\mathrm{al}^{24}$ and Matthies et $\mathrm{al}^{17}$ also found a lower alarm criterion, that is, a 35\% decrease in MEP amplitude, prone to trigger more warnings than with a 50\% decrease in MEP amplitude, in similar and larger groups of patients. This low alarm criterion is in line with the previous observation of the high rates of poor facial nerve outcomes in the cases with a $50 \%$ decrease in MEP amplitude. ${ }^{20,21}$ Unfortunately, these previous analyses of facial MEP performances were not enough acknowledged in the domain of intraoperative neuromonitoring.

Sensitivity and negative predictive values were found to be higher than those reported recently by Tawfik et al. ${ }^{19}$ This observation can be explained by the fact that a single alarm criterion was applied across different lesion types. This criterion

Table 2 Postoperative evaluation of sensitivities and of negative predictive values for different decreases in motor evoked potential (MEP) amplitudes, computed in the group of patients with vestibular schwannomas

\begin{tabular}{|l|l|l|l|l|l|}
\hline MEP decrease (\%) & True negative & True positive & False negative & Sensitivity (\%) & Negative predictive value (\%) \\
\hline $10^{\mathrm{a}}$ & 5 & 8 & 1 & 88.9 & 83.3 \\
\hline 20 & 6 & 8 & 1 & 88.9 & 85.7 \\
\hline 30 & 6 & 8 & 1 & 88.9 & 85.7 \\
\hline 40 & 6 & 7 & 2 & 77.8 & 75.5 \\
\hline 50 & 6 & 7 & 2 & 77.8 & 75.0 \\
\hline 60 & 6 & 6 & 3 & 66.7 & 66.7 \\
\hline 70 & 6 & 6 & 3 & 66.7 & 66.7 \\
\hline 80 & 6 & 5 & 4 & 55.5 & 60.0 \\
\hline 90 & 6 & 5 & 4 & 55.5 & 60.5 \\
\hline 100 & 6 & 5 & 4 & 55.5 & 60.0 \\
\hline
\end{tabular}

The numbers of true positive (TP; permanent MEP decrease above that threshold at the end of resection and new deficits), true negative (TN; MEP decrease under that threshold at the end of resection and no deficit), and false negative (FN; MEP decrease under that threshold at the end of resection but new deficits) are indicated with sensitivity $(T P /(T P+F N))$ and with negative predictive value $(T N /(T N+F N))$.

${ }^{a}$ With this criterion, one case would be unclassified (equivalent to one case of false positive). 


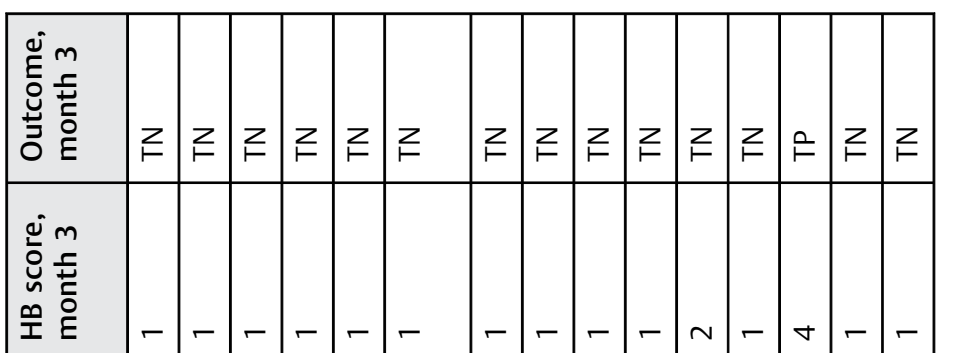

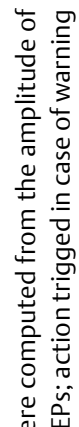

范

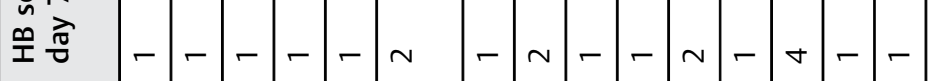

\ᄒ

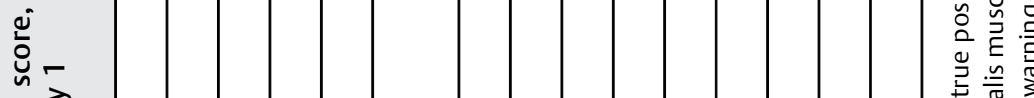

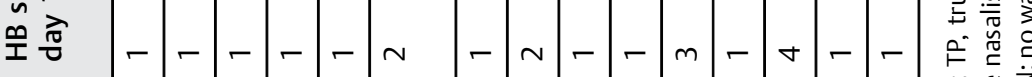

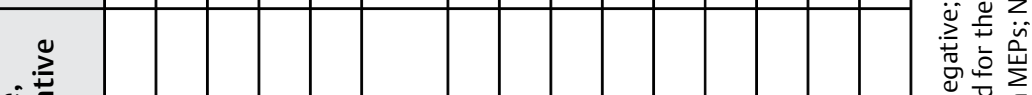

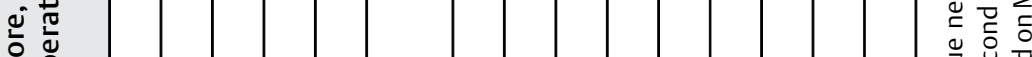

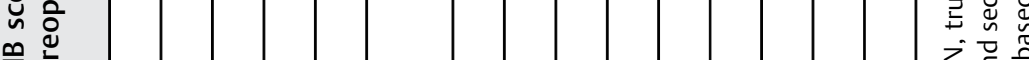

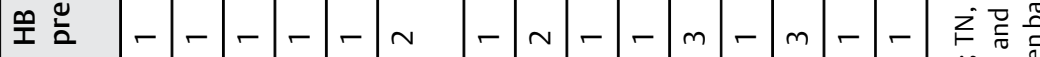

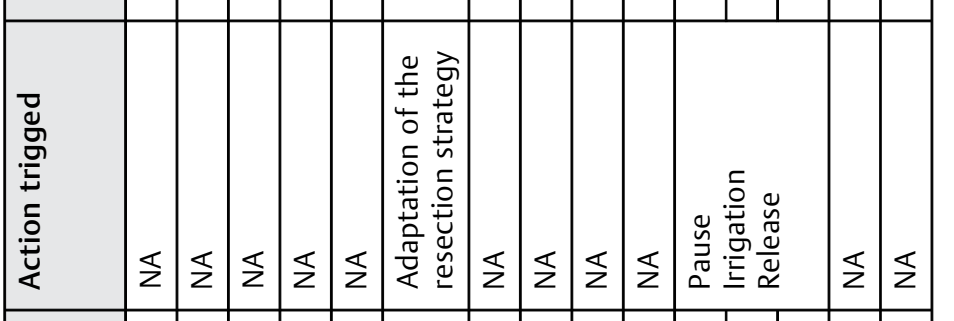

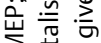

$\frac{z}{z}$
$\frac{5}{2}$
$\frac{\pi}{\alpha}$

$\bar{z}$

$\frac{\sqrt{2}}{E}$

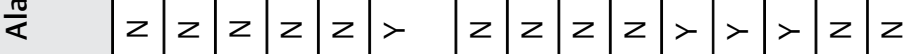

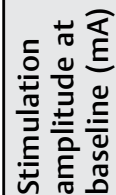

\begin{tabular}{llll|l|l|l|l|l|l|l|l|l|l|l|l} 
& & & & & & & & & & & & & \\
\hline
\end{tabular}

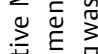

龸完

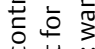

点玄

乙苞

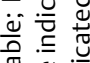

氜

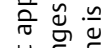

苍空高

$\sum_{i} \widehat{C}$

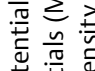

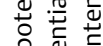

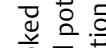

$\overline{\underline{n}} \frac{0}{\bar{n}}$

$\therefore=$

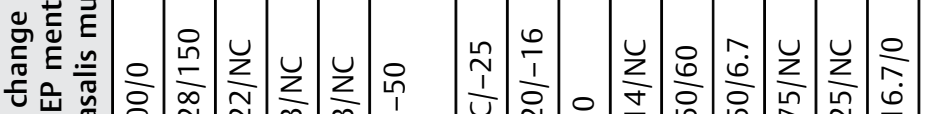

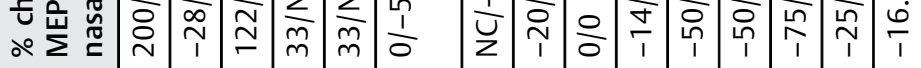

E

崖 $\frac{E}{0}$

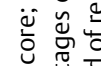

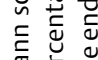

\begin{tabular}{|c|c|c|c|c|c|c|c|c|c|c|c|c|c|c|c|c|}
\hline 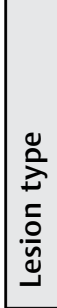 & 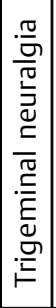 & 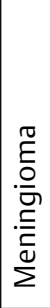 & 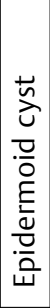 & 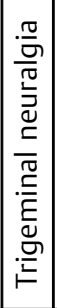 & 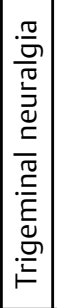 & 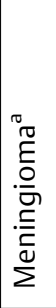 & 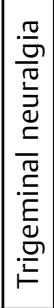 & 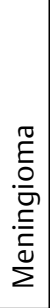 & 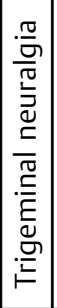 & 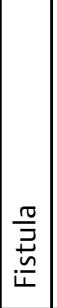 & 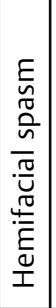 & 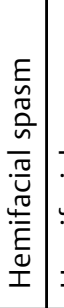 & 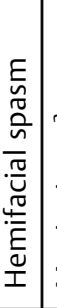 & 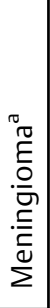 & 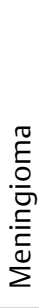 & 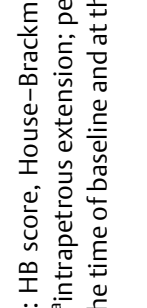 \\
\hline & $\frac{6}{a}$ & $\frac{\hat{a}}{2}$ & $\frac{\infty}{a}$ & 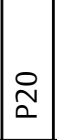 & $\overline{\widetilde{\alpha}}$ & $\stackrel{\tilde{a}}{\tilde{\alpha}}$ & $\stackrel{\mathbb{Z}}{\mathbb{a}}$ & $\stackrel{\tilde{\Omega}}{\widetilde{\alpha}}$ & 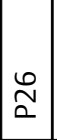 & $\hat{a}$ & $\stackrel{\infty}{\stackrel{\infty}{\Sigma}}$ & $\stackrel{\overparen{D}}{\underline{\Omega}}$ & 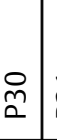 & $\bar{n}$ & & 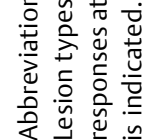 \\
\hline
\end{tabular}


Table 4 Postoperative evaluation of sensitivities and of negative predictive values for different decreases in motor evoked potential (MEP) amplitudes, computed in the group of patients with other cerebellopontine angle lesions

\begin{tabular}{|l|l|l|l|l|l|}
\hline MEP decrease (\%) & True negative & True positive & False negative & Sensitivity (\%) & Negative predictive value (\%) \\
\hline $10^{\mathrm{a}}$ & 6 & 1 & 0 & 100.0 & 100.0 \\
\hline $20^{\mathrm{a}}$ & 9 & 1 & 0 & 100.0 & 100.0 \\
\hline $30^{\mathrm{a}}$ & 12 & 1 & 0 & 100.0 & 100.0 \\
\hline $40^{\mathrm{a}}$ & 12 & 1 & 0 & 100.0 & 100.0 \\
\hline $50^{\mathrm{a}}$ & 15 & 1 & 0 & 100.0 & 100.0 \\
\hline $60^{\mathrm{a}}$ & 15 & 1 & 0 & 100.0 & 100.0 \\
\hline $70^{\mathrm{a}}$ & 15 & 1 & 0 & 100.0 & 100.0 \\
\hline 80 & 15 & 0 & 1 & 0.0 & 93.7 \\
\hline 90 & 15 & 0 & 1 & 0.0 & 93.7 \\
\hline 100 & 15 & 0 & 1 & 0.0 & 93.7 \\
\hline
\end{tabular}

The numbers of true positive (TP, MEP decrease above that threshold at the end of resection and new deficits), true negative (TN, MEP decrease under that threshold at the end of resection and no deficit) and false negative (FN, MEP decrease under that threshold at the end of resection but new deficits) are indicated with sensitivity $(T P /(T P+F N))$ and with negative predictive value $(T N /(T N+F N))$.

${ }^{a}$ With this criterion, cases would be unclassified (equivalent to false-positive cases).

was a 50\% decrease in MEP amplitude, which might not be low enough for vestibular schwannomas. Indeed, a decrease in MEP amplitude of more than $30 \%$ can already be associated with an increase in the HBS and hence with a facial nerve deficit. This alarm criterion could have led to a lower sensitivity, with FN cases, and could have contributed to the conclusion that neuromonitoring did not decrease the rate of facial nerve deterioration. ${ }^{19}$

Seidel et $\mathrm{al}^{16}$ reported a low complication rate using continuous direct electrical stimulation during the resection of vestibular schwannomas. In the present series, direct electrical stimulation was usually performed temporarily and was not always done before the observation of MEP decreases. The use of continuous direct electrical stimulation in our series could have contributed to better protecting the facial nerve of at least one accidental event and to further lowering the facial nerve deterioration rate.

Also, the fact that the alarm criterion at the time of the surgery was a $50 \%$ decrease in MEP amplitudes and not yet a reproducible $30 \%$ for vestibular schwannomas could have contributed to not giving early enough the information that continuing the resection would present a high risk of irreversible facial nerve deficits.

\section{Limitations}

This is a retrospective study in a group of patients of limited size. Larger group sizes would help narrower the confidence intervals.

For ethical reasons, no control group was included in the study as is the case for the majority of studies conducted in the domain of intraoperative neuromonitoring.

\section{Conclusion}

The analysis of facial nerve MEPs suggested that the most reliable alarm criterion to be applied was different for surgeries of vestibular schwannomas as compared with surgeries of other cerebellopontine angle lesions. As introduced by Dong et $\mathrm{al}^{24}$ and recalled by Matthies et $\mathrm{al}^{17}{ }^{17} \mathrm{a}$ reproducible $30 \%$ decrease in MEP amplitudes, resistant to a $10 \%$ increase in stimulation intensity and prone to trigger early warnings, was verified for vestibular schwannomas. Given the small size of our group with other cerebellopontine angle lesions, the established 50\% decrease in MEP amplitude as an alarm criterion should not be loosened to a less strict criterion for these surgeries. The stricter alarm criterion found for the resection of vestibular schwannomas, which is the highest risk surgery for the facial nerve, suggests that its application could improve the reliability of facial MEPs and render facial MEPs contributive to the decrease of the principal complications of this surgery.

\section{Conflict of Interest}

None.

\section{Acknowledgments}

The authors thank all patients who agreed to be part of the study. We thank D. Conan and $\mathrm{Ch}$. Bech who are either nurse or technician specializing in intraoperative neuromonitoring.

\section{References}

1 Al-Shudifat AR, Kahlon B, Höglund P, Lindberg S, Magnusson M, Siesjo P. A patient-assessed morbidity to evaluate outcome in surgically treated vestibular schwannomas. World Neurosurg 2016;94:544-550.e2

2 Nuño M, Ugiliweneza B, Boakye M, Monfared A. Morbidity of vestibular schwannomas as documented by treating providers. Otol Neurotol 2019;40(02):e142-e149

3 Agarwal V, Babu R, Grier J, et al. Cerebellopontine angle meningiomas: postoperative outcomes in a modern cohort. Neurosurg Focus 2013;35(06):E10

4 Khan SA, Khan B, Khan AA, et al. Microvascular decompression for trigeminal neuralgia. J Ayub Med Coll Abbottabad 2015;27(03): 539-542 
5 Lee MH, Jee TK, Lee JA, Park K. Postoperative complications of microvascular decompression for hemifacial spasm: lessons from experience of 2040 cases. Neurosurg Rev 2016;39(01):151-158, discussion 158

6 Liu SW, Jiang W, Zhang HQ et al. Intraoperative neuromonitoring for removal of large vestibular schwannoma: facial nerve outcome and predictive factors. Clin Neurol Neurosurg 2015;133:83-89

7 Kartush JM. Neurography for intraoperative monitoring of facial nerve function. Neurosurgery 1989;24(02):300-301

8 Kombos T, Suess O, Kern BC, Funk T, Pietilä T, Brock M. Can continuous intraoperative facial electromyography predict facial nerve function following cerebellopontine angle surgery? Neurol Med Chir (Tokyo) 2000;40(10):501-505, discussion 506-507

9 Yingling CD, Gardi JN. Intraoperative monitoring of facial and cochlear nerves during acoustic neuroma surgery. Otolaryngol Clin North Am 1992;25(02):413-448

10 Prell J, Strauss C, Rachinger J, et al. The intermedius nerve as a confounding variable for monitoring of the free-running electromyogram. Clin Neurophysiol 2015;126(09):1833-1839

11 Romstöck J, Strauss C, Fahlbusch R. Continuous electromyography monitoring of motor cranial nerves during cerebellopontine angle surgery. J Neurosurg 2000;93(04):586-593

12 Kartush JM, Niparko JK, Bledsoe SC, Graham MD, Kemink JL. Intraoperative facial nerve monitoring: a comparison of stimulating electrodes. Laryngoscope 1985;95(12):1536-1540

13 Silverstein $\mathrm{H}$. Microsurgical instruments and nerve stimulator: monitor for retrolabyrinthine vestibular neurectomy. Otolaryngol Head Neck Surg 1986;94(03):409-411

14 Delgado TE, Bucheit WA, Rosenholtz HR, Chrissian S. Intraoperative monitoring of facila muscle evoked responses obtained by intracranial stimulation of the facila nerve: a more accurate technique for facila nerve dissection. Neurosurgery 1979;4(05): 418-421

15 Cornelius JF, Schipper J, Tortora A, et al. Continuous and dynamic facial nerve mapping during surgery of cerebellopontine angle tumors: clinical pilot series. World Neurosurg 2018;119:e855-e863

16 Seidel K, Biner MS, Zubak I, Rychen J, Beck J, Raabe A. Continuous dynamic mapping to avoid accidental injury of the facial nerve during surgery for large vestibular schwannomas. Neurosurg Rev 2018

17 Matthies C, Raslan F, Schweitzer T, Hagen R, Roosen K, Reiners K. Facial motor evoked potentials in cerebellopontine angle surgery: technique, pitfalls and predictive value. Clin Neurol Neurosurg 2011;113(10):872-879

18 Tokimura H, Sugata S, Yamahata H, Yunoue S, Hanaya R, Arita K. Intraoperative continuous monitoring of facial motor evoked potentials in acoustic neuroma surgery. Neurosurg Rev 2014;37 (04):669-676

19 Tawfik KO, Walters ZA, Kohlberg GD, et al. Impact of motorevoked potential monitoring on facial nerve outcomes after vestibular schwannoma resection. Ann Otol Rhinol Laryngol 2019;128(01):56-61

20 Acioly MA, Gharabaghi A, Liebsch M, Carvalho $\mathrm{CH}$, Aguiar PH Tatagiba M. Quantitative parameters of facial motor evoked potential during vestibular schwannoma surgery predict postoperative facial nerve function. Acta Neurochir (Wien) 2011;153 (06):1169-1179

21 Fukuda M, Oishi M, Hiraishi T, Saito A, Fujii Y. Intraoperative facial nerve motor evoked potential monitoring during skull base surgery predicts long-term facial nerve function outcomes. Neurol Res 2011;33(06):578-582

22 Howick J, Cohen BA, McCulloch P, Thompson M, Skinner SA. Foundations for evidence-based intraoperative neurophysiological monitoring. Clin Neurophysiol 2016;127(01):81-90

23 Verst SM, Sucena AC, Maldaun MV, Aguiar PH. Effectiveness of C5 or C6-Cz assembly in predicting immediate post operative facial nerve deficit. Acta Neurochir (Wien) 2013;155(10):1863-1869

24 Dong CC, Macdonald DB, Akagami R, et al. Intraoperative facial motor evoked potential monitoring with transcranial electrical stimulation during skull base surgery. Clin Neurophysiol 2005; 116(03):588-596

25 House JW, Brackmann DE. Facial nerve grading system. Otolaryngol Head Neck Surg 1985;93(02):146-147

26 Boex C, Haemmerli J, Momjian S, Schaller K. Prognostic values of motor evoked potentials in insular, precental, or postcentral resections. J Clin Neurophysiol 2016;33(01):51-59 